\title{
O SUPERCONTINENTE NEOPROTEROZÓICO E AS INTERAÇÕES GONDWANA - LAURENTIA DURANTE O PALEOZÓICO INFERIOR - MÉDIO
}

\author{
Tradução do Original em espanhol "realizada com a permissão dos autores" por Benjamim B. Brito Neves***
}

\author{
LUÍS H. DALLA SALDA* \& IAN W.D. DALZIEL**
}

\begin{abstract}
THE NEOPROTEROZOIC SUPERCONTINENT AND THE GONDWANA LAURENTIA INTERACTIONS DURING THE LOWER-MID PALEOZOIC. Gondwana-Laurentia paleomagnetic reconstructions and geological fits are presented, from the Neoproterozoic to the Devonian. They show the breakup of a supercontinent, the origin of the southern lapetus, an early Paleozoic South America active margin, and Laurentia trailing edge; before Taconian Laurentia - Gondwana collision. Following it, the Acadian transpression type orogeny took place. The paleogeography supports the idea of a common origin for the Appalachian - Famatinian orogenic belts, in both Laurentia and South America.
\end{abstract}

Keywords: Neoproterozoic supercontinent, Laurentia, South America, Lower-Mid Paleozoic, Paleogeography, çollision.

RESUMO São apresentadas reconstruções paleomagnéticas e correlações geológicas do GondwanaLaurentia, desde o Neoproterozóico até o Devoniano. Elas mostram a ruptura de um supercontinente, a origem do lapetus do Sul, uma margem ativa para o Eopaleozóico da América do Sul, e a margem passiva de Laurentia, antes da colisão lacónica Laurentia-Gondwana. Posteriormente a ela, ocorreu o episodio orogênico tipo transpressivo acádico. A paleogeografia avaliza a ideia de origem comum para os Cinturões Orogênicos Apalachiano e Famatiniano, em Laurentia e América do Sul, respectivamente.

Palavras-chave: Supercontinente neoproterozóico, Laurentia, América do Sul, Paleozóico Inferior-Médio, Paleogeografia, colisão.

INTRODUÇÃO A reunião da América do Sul - Laurentia em um supercontinente do Pré-Cambriano Superior, com um embasamento comum de consolidação Grenville, é respaldada por dados paleomagnéticos, associada aos demais dados geológicos e radiométricos (Dalziel 1991, Dália Salda et al 1992a, 1992b, 1992c, Dalziel 1992a, 1992b, Dalziel \& Gahagan 1992, Dalziel et al 1992, Dalziel \& Grunow 1992, Dália Salda et al 1993, Dalziel et al 1993). No norte do Chile, complexos metamórficos incluem lavas com idade Sm-Nd de 1.460 Ma, migmatitos com idades Sm-Nd de $1.214 \mathrm{Ma}$, assim como componentes crustais herdados de idade U-Pb de $777 \mathrm{Ma}$ em rochas neopaleozóicas (Damn et al 1990, Baeza \& Pichowiak 1981). A Serra de Pie de Paio conta com idade $\mathrm{Rb} / \mathrm{Sr}$ de 1.027 Ma (Varela \& Dália Salda 1993). Zircões, com idades em torno de $1.100 \mathrm{Ma}$, em rochas paleozóicas da Pré-CordiIheira (Loske 1992) apontam uma idade "grenvilliana" para o embasamento sotoposto. Metamorfitos da Patagônia distinguem o chamado terreno Coluhuincul (900 - $900 \mathrm{Ma}$ ), cujas idades Sm-Nd indicam que seu protólito se instalou na crosta entre 1.250 e 800 Ma (Dália Salda et al 1990). Gnaisses dessa mesma região indicaram idades de 1.190 e 1.200 Ma (Linares et al 1988). Estes últimos dados foram responsáveis pela sugestão do "Terreno Occidentália", précambriano, colocado a oeste do extremo sul da América do Sul (Dália Salda et al 1992a, b e c).

\section{PALEOGEOGRAFIA: DA RUPTURA DE UM SUPER- CONTINENTE AS COLISÕES LAURENTIA-AMERI-}

CA DO SUL Na figura 1, observa-se a reconstrução (projeção ortográfica) do Supercontinente LaurentiaGondwana $c a$. $750 \mathrm{Ma}$, considerando o rifteamento do oeste de Laurentia com Antártica Oriental - Austrália, a abertura do Oceano Pacífico e a aglutinação de Gondwana (Dalziel 1991, Hoffman 1991, Dalziel 1992a, b).
O promontório de Labrador - Groenlândia de Laurentia e o cotovelo de Arica do Cráton Sul-Americano são usados para o arranjo destas massas continentais (Dalziel 1992b); Báltica se ajustou adjacente à Groenlândia e a noroeste da América do Sul, com a orientação relativa a Laurentia, como proposto por Gower et al (1990). O supercontinente se mostra no marco de referência do pólo paleomagnético de Laurentia (SP - Pólo Sul), determinado por Symons \& Chaisson (1990). O círculo NAM é de 95\% de confiança. Sibéria está localizada de acordo com a sugestão de Hoffmann (1991), mas pode ter derivado de Laurentia nesta época (e.g., Heaman et al 1992).

$\mathrm{Na}$ reconstrução do Cambriano Médio (ca. $530 \mathrm{Ma}$, Fig. 2), foram utilizados os mesmos pontos de Laurentia, Gondwana e Báltica de McKerrow et al (1992), aqueles pólos de Kirschvink (1978) para Gondwana, Tanczyk et al (1987) para Laurentia, e os de Frendi \& Torsvik (1991) para Báltica, sendo este último usado como pólo Sul (SP = Pólo Sul).

Este episódio extensional inicia-se com o rifteamento do supercontinente (separação total de Laurentia e América do Sul) com o franco desenvolvimento de bacias, tal como representada pela Formação Puncoviscana, na qual lavas básicas alcalinas ascenderam à crosta (Omarini \& Alonso 1987, Coira et al 1990). Interpreta-se que este episódio atingiu extensão litosférica de vulto que evoluiu e originou o sul do Oceano lapetus. Laurentia derivou da América do Sul, desenvolvendo plataforma carbonática em sua borda oriental durante o Cambriano.

É importante destacar o papel da Pré-Cordilheira Paleozóica Inferior do oeste argentino, parte do Terreno Occidentália (OT., Fig. 2 e 4). Como já fora anteriormente mencionado (e.g., Ramos et al 1986), a estratigrafia e o conteúdo faunistico do banco carbonático da mesma idade nos Apalaches são surpreendentemente similares. A. Palmer

\footnotetext{
* Centro de Investigaciones Geológicas, Universidade Nacional de La Plata, Calle 1, 644, 1900 La Plata, Argentina *** University of Texas at Austin, Institute for Geophysics, 8701 Mopac Boulevard, Austin, Texas 78759 - 8397, USA
} 


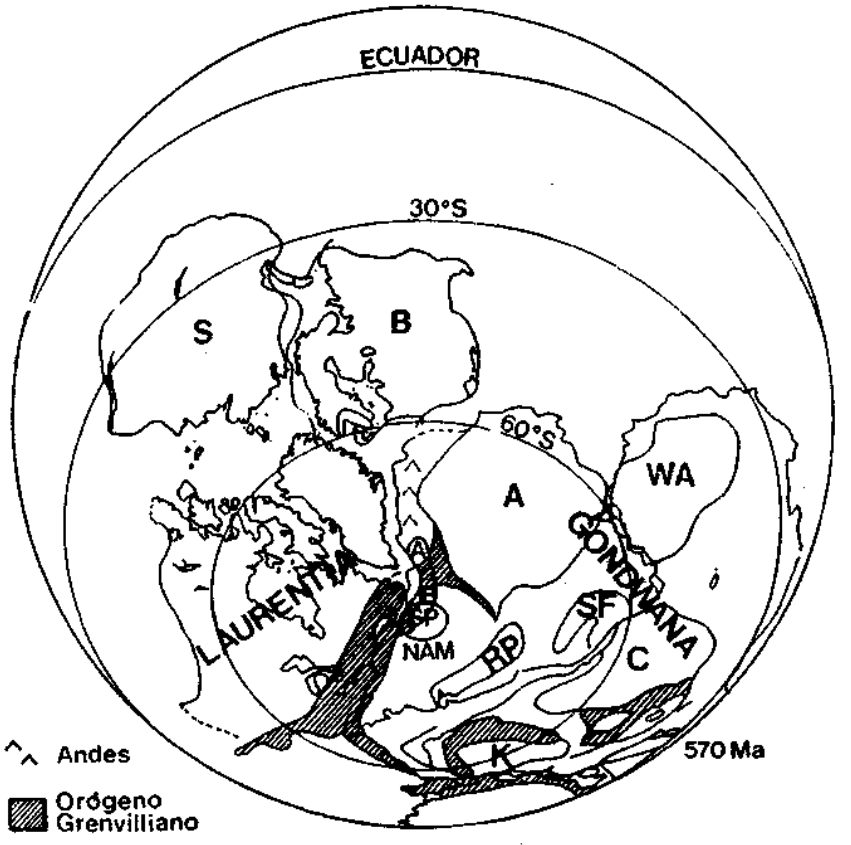

Figura 1. - Reconstrução (projeções ortográficas) do Supercontinente Neoproterozóico, incluindo Laurentia e Gondwana (ca. 570 Ma). A. Cráton Amazônico; AB. Maciço Arequipa-Belém; B. Báltica; $\boldsymbol{C}$. Cráton do Congo; K. Cráton do Kalahari; RP. Cráton Rio de La Plata; S. Sibéria; SR Cráton do São Francisco; WA. Cráton do Oeste África, segundo Dalziel et al. (1993); NAM. Laurentia; SP. Pólo Sul. Figure 1 - Reconstruction (orthograhic propections) of the neoproterozoic supercontínent including Laurentia and Gondwana (ca. $570 \mathrm{Ma}$ ). A. Amazónia Cráton; AB. Arequipa-Belém massif; B. Báltica; C. Gongo Cráton; K. Kalahari Cráton; RP. São Francisco Cráton; WA. West África Cráton; according to Dalziel et al. (1993); NAM. Laurentia; SP. South Polé

(comunicação pessoal) recentemente informou que é possível correlacionar, com poucas dúvidas, o Cambriano-Tremadociano da Pré-Cordilheira com equivalentes da plataforma carbonática do leste de Laurentia.

Simultaneamente, a leste da dorsal do Oceano lapetus do Sul (D, Fig. 2), a margem ocidental da América do Sul foi ativa (MA, Fig. 2). Nessa margem, desenvolveu-se um arco magmático (granitóides do Ciclo Pampeano, segundo Acenola \& Toselli 1976) que apresenta afinidades toleíticas (Jezek et al 1987, Rapela et al 1990, 1992), que já foram interpretados como pré-colisionais e relacionados à subducção (Gallinsky \& Miller 1989). A interpretação é de que nesta instância do desenvolvimento, arcos-de-ilhas foram acrescionados à magem ativa àquele descrito por Vujovich (1992), nas Serras Pampeanas Ocidentais.

A leste do banco carbonático dos Apalaches e da sutura do lapetus, são encontrados granitóides, assim como a crosta continental que os contém; os arcos-de-ilhas antes mencionados são considerados parte da crosta da América do Sul, que ficou acrescionada a Laurentia durante a Orogenia Tacônica.

A paleogeografia, para os tempos das Orogenias Tacônica e Shakleton (Fig. 3), realizou-se utilizando os pólos de Van Der Voo (1988) para Laurentia e Gondwana, aos 487 Ma, e Torsvik et al (1990) para Báltica.

Os eventos colisionais entre Laurentia Oriental e Gondwana Ocidental, referidos às Orogenias Tacônica e Acádica (Figs. 2 e 4), são sugeridos como a causa e origem comum dos Orógenos Apalachiano e Famatiniano. Esses episódios orogênicos também estão relacionados a colisões, baseando-se em estudos detalhados das rochas granitóides (Rapela et al 1992). É possível uma extensão ao continente

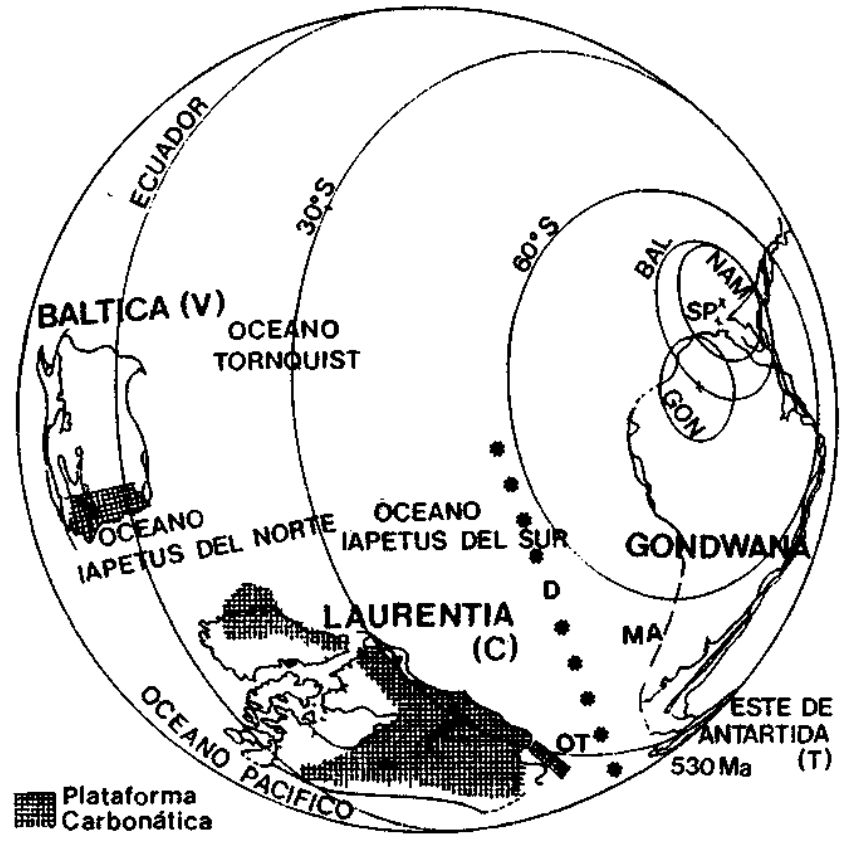

Figura 2 - Reconstrução para o Cambriano (ca. 530 Ma). Os círculos de confiança (nivel 95\%) aparecem como: $\boldsymbol{B A L}$. Báltica; GON. Gondwana; NAM. Laurentia; E. Montanhas Ellsworth (posição de Dalziel \& Gronow 1992); OT. Terrenos Occidentália (inclui Pré-Cordilheira) da América do Sul, e restaurando as Serras de Ouachita de Laurentia, como sugerido por Dália Salda et al. (1992b); Ma. Margem Ativa da América do Sul; D. Dorsal do lapetus do Sul; PR. Serras Pampeanas; V, C e T. Disposição das províncias de trilobitas de Jeil (1974), segundo Dalziel et al. (1993) Figure 2 - Reconstruction for the Cambrian Period (ca. $530 \mathrm{Ma}$ ). Trustful circles (about 95\%) are assigned as: BAL. Báltica; GON. Gondwana; NAM. Laurentia; E. Ellsworth mountains (position according to Dalziel \& Gronow 1992). OT. Occidentália terrane (só including Pré-Cordilheira) for South America, and the Ouachita mountains are restored according with the suggestion of Dália Salda et al. (1992b); MA. active rnargin of South America; D. lapetus dorsal; PR. Sierras Pampeanas; V, C and T. localization of the trilobites provinces of Jeil (1974); after Dalziel et al. (1993)

antártico desses eventos através do Cinturão Orgênico Ross (Dália Salda et al. 1992b, Dalziel et al. 1993).

O orógeno Famatiniano desenvolveu-se de Arequipa a Patagônia, que é interpretado como resultado inicial da colisão lacónica Laurentia x América do Sul. Um dos dados que sustentam esta ideia é a presença do Terreno Occidentália (Dália Salda et al 1992a, b, c). Esse terreno, que ocorre na direção norte-sul, dispõe-se a oeste do Orógeno Famatiniano. Em sua maior parte, há evidências de embasamento com rochas metamórficas pré-cambrianas, algumas delas remobilizadas. Também seriam parte de Occidentália a região Pré-Cordilheira de Cuyo, e o terreno chamado "Chilenia" (Ramos et al 1986, Ramos 1988). Occidentália foi interpretado com uma relíquia de Laurentia Oriental (nas coordenadas atuais) anexada à borda sudoeste de Gondwana, durante o fechamento do oceano lapetus Sul, e a conseguinte colisão entre Laurentia e Gondwana (Dália Salda et al 1992a, b, c), durante o Ordoviciano (Tacônica, ca. 440 - $480 \mathrm{Ma}$ ).

No oeste da Pré-Cordilheira, desenvolveu-se, durante o Llandeiliano-Caradociano, uma bacia tipo rifte intracontinental, considerando o Paleozóico Inferior da Pré-Cordilheira como parte da talude continental (associado com os ofiolitos Caradocianos) como um reflexo da sedimentação em uma margem continental com um oceano a oeste (Ramos 


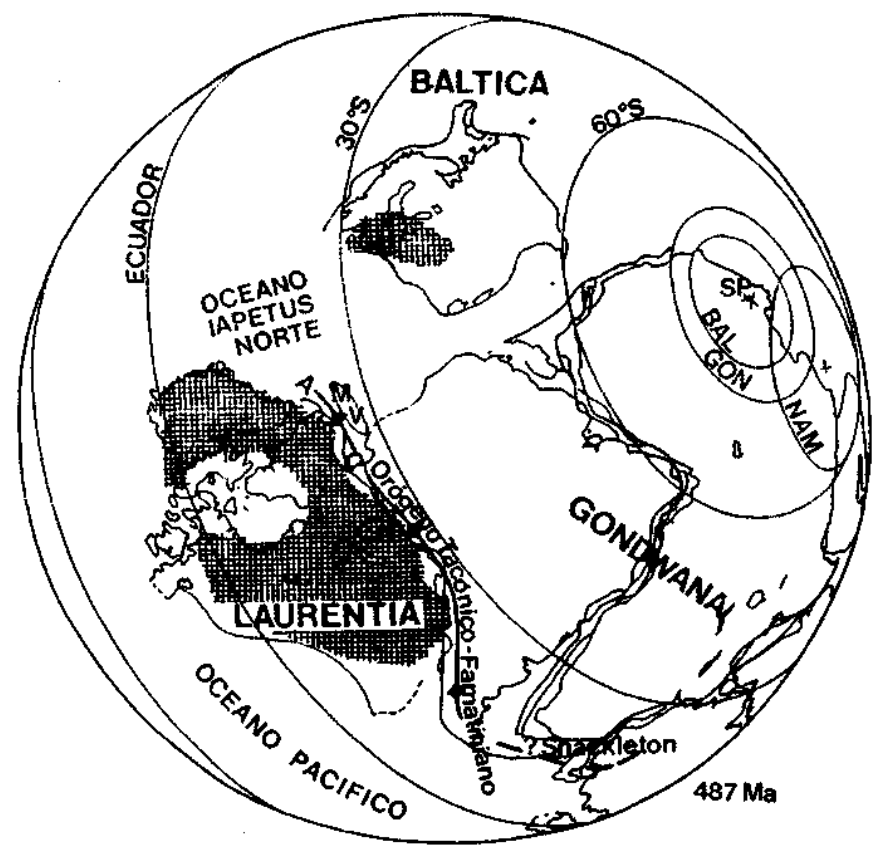

Figura 3 - Reconstrução para os tempos da Orogenia Tacônica (Ocloyica). Os círculos de confiança (nível 95\%) são denominados como na figura 2. AO. Orógeno Atholliano de McRerrow et al. (1991); MV. Terreno do Vale de Midland, segundo Dalziel et al, 7993

Figure 3 - Reconstruction for the Taconic (Ocloyic) Orogeny times. Trustful circles (about 95\%) assigned as figure 2, above: AO. Athollian Orogen of McKerrow et al. (1991); MV. Midland Valley terrane after Dalziel et al. (1993)

et al. 1986, Ramos 1988). Aparentemente, esta interpretação é oposta a que mostra o Orógeno Tacônico dos Apalaches. Não obstante, entende-se que os níveis do oeste da Pré-Cordilheira, relacionados com águas profundas, parecem ser - em parte - ou mais jovens que o Tremadociano, ou alóctones (transportados por falhamentos vindos do oeste). O rifteamento com os ofiolitos Caradocianos foram recentemente explicados como parte da evolução das montanhas Ouachitas do sudeste de Laurentia (Dália Salda et al 1992b).

Posteriormente, entende-se que a Orogênese Acádica (Fig. 4) seguiu a separação Laurentia-Gondwana pósTacônica.

Estima-se que essa orogênese originou-se durante um episódio orogênico transpressivo dextral entre continentes (Dalziel et al. 1993, ver também a reconstrução de Kent e Van Der Voo 1990). Este novo episódio colisional oblíquo, rotacional no sentido horário de Laurentia contra o oeste da América do Sul, causou a deformação das bacias pré-Acádicas (Ordoviciano Superior a Devoniano Médio) e elevou substancialmente o fluxo térmico das faixas continentais em contato, produzindo metamorfismo (ou o rejuvenescimento de rochas mais antigas) e a colocação de granitos sin a póscolísionais. Rapela et al. (1992), baseados no estudo detalhado de granitóides dessa idade, também sugeriram sua origem colisional.

CONCLUSÕES A evolução paleogeográfica do oeste do supercontinente neoproterozóico mostra um episódio de ruptura que deu origem ao Oceano lapetus do Sul. Enquanto Laurentia derivava formando uma plataforma carbonática Cambriana-Tremadociana (Plataforma pré-Tacônica nos Apalaches e sua continuação austral na Pré-Cordilheira de

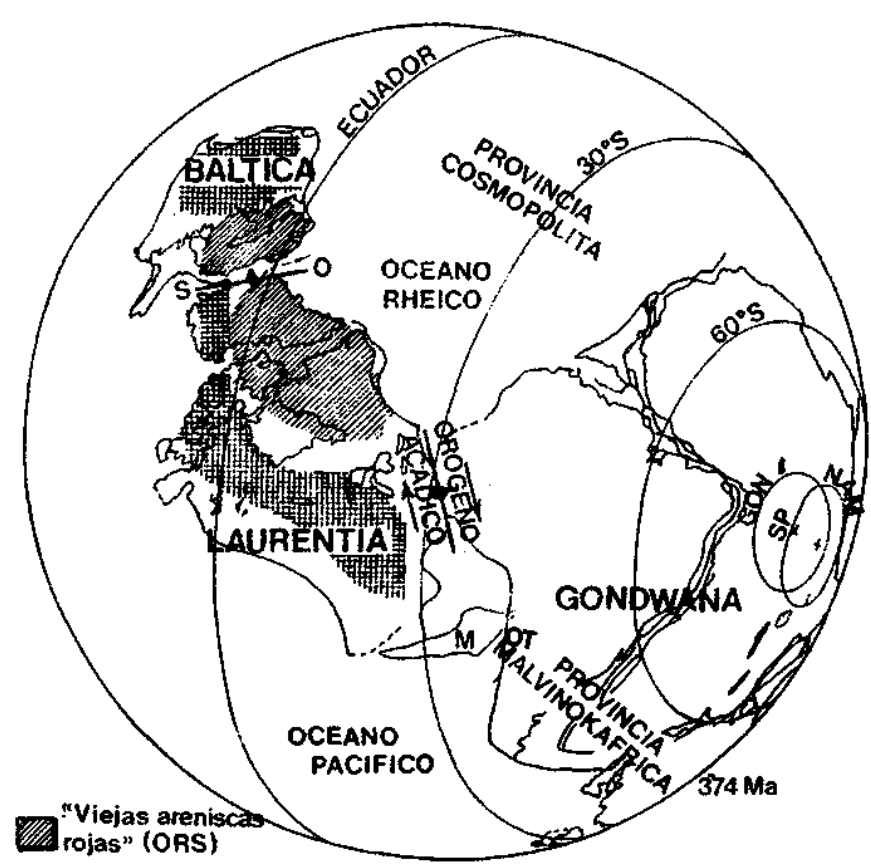

Figura 4 -Reconstrução (ca. 374 Ma), usando os pólos de Van Der Voo (1988). Os círculos menores (95\% de confiança), denominados como na figura 2. SO. Orógeno Escandinavo; M. México; OT. Terreno Occidentalia, acrescionado na colisão Tacônica

Figure 4 - Reconstrucion (ca. $374 \mathrm{Ma}$ ) according with Van Der Voo (1988) polés. Minor circles (trustful about 95\%) assigned as in the figure 2 SO. Scandinavian orogen; M. México; OT. Occidentalia Terrane, accreted during the Taconic collision

Cuyo), o oeste da América do Sul foi uma borda ativa. Ali, a subducçao da placa oceânica ocidental do lapetus desenvolveu um arco tipo andino (granitóides pampeanos na América do Sul e nos Apalaches). Posteriormente (ca. 480-460 $\mathrm{Ma}$ ), Laurentia e Sul-América colidiram, originando o Orógeno Tacônico. As faixas sincrônicas ígneas e metamórficas dos Apalaches e do Orógeno Famatiniano dão uma ideia da magnitude dessa colisão. Em síntese, a paleogeografia aqui apresentada e a continuidade dos Apalaches como um "par geotectônico" sincrônico na América do Sul uma plataforma carbonática paleozóica inferior a oeste, e um cinturão ígneo-metamórfico paleozóico inferior-médio a leste do mesmo (com relíquias de rochas pré-cambrianas) dá respaldo à hipótese de que o Orógeno Famatiniano foi a extensão sul dos Apalaches.

Interpreta-se que a crosta continental de Laurentia, a leste dos Apalaches, corresponde a um terreno complexo alóctone, de origem sulamericana, integrado pelo arco magmático cambriano (Pampeano), pré-colisional, e as faixas metamórficas e plutões colisionais (principalmente do Tacônico). Enquanto, na América do Sul, Occidentalia corresponde a uma relíquia de Laurentia, composta por um embasamento de idade Grenville e uma plataforma carbonática do Paleozóico Inferior. Ambos os terrenos permanecem acrescionados a Laurentia e América do Sul com a colisão Tacônica. Sugere-se que, durante o rift-drift pré-Acadiano, o Orógeno Famatiniano-Apalachiano foi segmentado, deixando parte do mesmo em Laurentia e outra parte na América do Sul.

Agradecimentos Este trabalho foi realizado com subsídios do CONICET 30021-30069 e da National Science Foundation DPP 8917127. As reconstruções foram realizadas usando o programa "PLATES" no Institute for 
Geophysics, na Universidade do Texas, em Austin. Agradecemos particularmente a 1. Gahagan, C. Cingolani e R. Varela. Ainda agradecemos a A. Grunow, A. Plamer, R.
Hatcher Jr., L. Sapalletti, R. Trompette e E. Aragón, por seus aportes, opiniões e críticas construtivas. Este trabalho é uma contribuição aos Projetos IGCP 345, 270288.

\section{REFERENCIAS BIBLIOGRÁFICAS}

ACENOLA, G. \& TOSELLI, J. 1976. Consideraciones estratigráficas y tectónicas sobre el Paleozóico Inferior dei noroeste argentino. In: CONGR. LATINOAMER. GEOL., 2. Caracas, 1976. Actas... Caracas, v. 2, p. 755-763

BAEZA, L. \& PICHOWIAK, S. 1988. Ancient crystalline basement provinces in the north Chilean Andes-Relicts of continental crust development since the mid-Proterozoic. In: B AHLBUR, G. \& GIESE, C.H. eds. The Southern Central Andes. Berlin, Springer-Verlag. p. 124. (Lecture Notes in Earth Sciences 17).

COIRA, B.; MANCA, N. CHAYLE, W. 1990. Registros volcánicos en Ia Formación Puncoviscana. In: ACENOLAZA, F; MILLER H.; TOSELLI, J. eds. El Ciclo Pampeano en el Noroeste Argentino. Tucumán, Universidad Nacional de Tucumán. (Série Correlación Geológica 4).

DALLA SALDA, L.; CINGOLANI, C.; VARELA, R. 1990. The origin of Patagonia. Rev. Comunicaciones, 41:53-64.

DALLA SALDA, L.; CINGOLANI, C.; VARELA, R. 1992a. Early Paleozoic orogenic belt of the Andes in southwestern South America: result of Laurentia - Gondwana collision? Geology, 20:617-620.

DALLA SALDA, L.; DALZIEL, L; CINGOLANI, C.; CINGOLANI, C.; VARELA, R. 1992b. Did the Taconic Appalachian continue into southern South America? Geology, 20:1059-1062.

DALLA SALDA, L.; CINGOLANI, C.; VARELA, R. 1992c. El orógeno colisional paleozóico en Argentina. In: TOSELLI, J. ed. El Paleozóico Inferior en Latinoamerica y Ia Génesis el Gondwana. Tucumán, Universidad Nacional de Tucumán. p. 165-169. (Série Correlación Geológica 9).

DALZIEL, I. 1991. Pacific margins of Laurentia and East AntarticaAustralia as a conjugate rift pair: evidence and implications for an Eocambrian supercontinent. Geology, 19:598-601.

DALZIEL, I. 1992a. On the organization of American Plates in the Neoproterozoic and the breakout of Laurentia. GSA Today, $11(2): 239-241$

DALZIEL, I. 1992b. Antactica; a tale of two supercontinents? Ann. Rev. Earth Planei. Sei. Lett., 20:501-526.

DALZIEL, I. \& GAHAGAN, L. 1992. North America as an exotic terrane and the origin of the Appalachian and Andean Mountain belts. 29th. In: INTER. GEOL. CONGR. Kioto, 1992. Abstracts... Kioto, v. II -1-4, 0-9, p. 4422

DALZIEL, L; DALLA SALDA, L.; GAHAGAN, L. 1992. North America as an "Exotic Terrane" and the origin of the Appalachian-Andean Mountain System. GEOL. SOC. AMER. Cincinnati, 1992. Abstracts... Cincinnati, Geol. Soe. Am. v. 7, p. 15055.

DALZIEL, I. \& GRUNOW, A. 1992. Late Fondwanide tectonic rotations within Gondwanaland. Tectonics, 11:603-606.

DALZIEL, L; DALLA SALDA, L.; GAHAGAN, L. 1993. LaurentiaGondwana interaction and the origin of the Appalachian-pre-Andean mountain system. Geol. Soe. Am. Buli., (in press).

DAMM, K; PICHOWIAK, S.; HARMON, S.; TOD, W.; KELLEY, S.; OMARINI, R.; NIEMEYER, H. 1990. Pre-Mesozoic evolution of the Central Andes; The basement revisited. In: Plutonism from Antárctica to Alaska. In: KAY, S. \& RAPELA, C. eds. Geol. Soe. Am. Spec. Paper, 241:101-126.

GALLISKY, M. \& MILLER, C. 1989. Petrogénesis de Ias trondhjemitas de Cachi: condicionamientos impuestos por elementos de tierras raras e implicâncias tectónicas. In: CINGOLANI, C. ed. Montevideo, Universidad de Montevideo. Reunión geotransectas America dei Sur, $1: 58-62$.

GOWER, C.; RIVERS, T.; RYAN, W. 1990. Mid-Proterozoic LaurentiaBáltico. Toronto, Geol. Assoe. Can. 581 p. (Sp. Paper. 38).

HEAMAN, L. LECHEMINANT, A; RANINBIRD, R. 1992. Nature and timing of Frankling igneous events, Canada: implications for a Late Proterozoic mantle plume and the break-up of Laurentia. Earth Planet. Sei. Lett., 109:117-131.

HOFFM AN, P. 1991. Did the breakout of Laurentia turn Gondwanaland inside out? Science, 252:1409-1412.

JELL, P. 1974. Faunal provinces and possible planetary reconstruction of the MiddleCambrian.y. Geol., 82:319-350.

JEZEK, P; LOTTNER, U.; MILLER, H.; WILLNER, A. 1987. La evolución geológica dei NW argentino entre el Precámbrico Superior y el término dei ciclo Pampeano. In: MILLER, H. ed. Investigaciones Alemanas
Recientes en Latinoamerica. Stuttgart, Nãgele U. Obermiller. p. 63-70. (Geologia).

KTRSCHVINK, J. 1978. The Precambrian-Cambrian boundary problem, paleomagnetic directions from the Amadeus Basin, central Austrália. Earth Planet. Sei. Lett., 40:91-100.

LINARES, E.; CAGNONI, M.; CAMPO, M.; OSTERA, O. 1988. Geochronology of metamorphic and eruptive rocks of southeastern Neuquen and northwestern Rio Negro Provinces, Argentine Republic. J. South Am. Earth Sei., 1:53-61.

LOSKE, W. 1992. The west-Argentinian Precordillera: A lower Paleozoic back are basin? In: GUTIERREZ MARCO, J.; SAAVEDRA, J.; RÁBANO, J. eds. Paleozóico Inferior de íbero-América. Mérida, Universidad de Extremadura. p. 96-97.

MCKERROW, W.; DEWEY, J.; SCOTESE, C. 1991. The Ordovician and Silurian developement of the lapetus ocean. Spec. Pap. Paleontol, 44:165-178.

MCKERROW, W; SCOTESE, C.; BRASIER, W. 1992. Early Cambrian continental reconstructions. /. Geol. Soe. London, 140:599-606.

OMARINI, R. \& ALONSO, R. 1987. Lavas en Ia Formación Puncoviscana, Rio Blanco, Salta, Argentina. In: CONG. GEOL. ARG., 10. Tucumán, 1987. Actas... Tucumán, Asoc. Geol Argent. v. 4 , p. $292-295$.

RAMOS, V. 1988. Late Proterozoic-Early Paleozoic of South America: a collisional history. Episodes, 11:168-175.

RAMOS, V; JORDAN, T.; ALLMENDINGER, R.; MPODOZIS, C.; KAY, S.; CORTÊS, J.; PALMA, M. 1986. Paleozoic terranes of the central Argentine-Chilean Andes. Tectonics, 5:855-880.

RAPELA, C.: TOSELLI, A · HEAMAN, L. SAAVEDRA, J. 1990. Granite plutonism of the Sierras pampeanas: an inner cordilleran Paleozoic are in the southern Andes. In: KAY, S. \& RAPELA, C. eds. Plutonism from Antárctica to Alaska. Geol. Soe. Am. Spec. Paper, 241:77-90.

RAPELA, C.; COIRA, B.; TOSELLI, A.; SAAVEDRA, J. 1992. El magmatismo dei Paleozóico Inferior en el Sudoeste de Gondwana. In: GUTIERREZ MARCO, J.; SAAVEDRA, J.; RABANO, J. eds. Paleozóico Inferior de íbero-América. Extremadura, Universidad de Extremadura. p. 21-68

SINHA, K.; HUND, E.; HOGAN, J. 1989. Paleozoic accretionary history of the North American Plate Margin (Central and Southern Appalachians): Constrains from the age, origin and distribution of granitic rocks. In: WILLHOSE, J. ed. Deep Structure and Past Kinematics of Accreted Terranes. Washington, IUGS. v. 5, p. 219-238. (Monography 50).

SYMONS, D. \& CHIASSON, A. 1990. Paleomagnetism of the Callander complex and the Cambrian apparent polar wander path for North America. Can. J. Earth Sei., 28:355-363.

TANCZYK, E.; LAPOINTE, L.; MORRIS, W.; SCHMIDT, P. 1987. A paleomagnetic study of the layered mafic intrusion at Sept-Iles, Quebec. Can. J. Earth Sei., 24:1431-1438.

TORSVIK, T.; OLESEN, O.; RYAN, P; TRENCH, A. 1990. On the paleogeography of Baltica during the Paleozoic: New paleomagnetic data from the Scandinavian Caledonides. Geophys. J. Int., 103:261-279.

TRENCH, A. \& TORSVIK, T. 1991. A revised Paleozoic polar wander path for southern Britain (eastern Avalonia). Geophys. J. Int. 104:227-233.

VAN DER VOO, R. 1988. Paleozoic paleogeography of North America, Gondwana, and intervening displaced terranes: comparison of paleomagnetism with paleoclimatology and biogeographycal patterns. Geol. Soe. Am. Buli., 100:311-324.

VARELA, R. \& DALLA SALDA, L. 1993. Geocronologia Rb-Sr de metamorfitas y granitoides dei tercio sur de Ia Sierra de Pie de Paio, San Juan, Argentina. Asoc. Geol. Argent. Rev. 47(3):271-276.

VUJOVICH, G. 1992. Estúdio Petrológico y Tectónico dei Metamorfismo dei Sector Occidental de Ia Sierra de Ia Huerta, Provinda de San Juan. La Plata. p. 238. (Tesis Doctoral, Universidad Nacional de La Plata).

MANUSCRITO A797

Recebido em 31 de agosto de 1993 Revisão do autor em 3 de marco de 1994 Revisão aceita em 16 de maio de 1994 